MATEMATIKA, 2018, Special Issue, 179-187

(C) Penerbit UTM Press. All rights reserved

\title{
Transcritical Flow Over a Bump using Forced Korteweg-de Vries Equation
}

\author{
${ }^{1,3}$ Vincent Daniel David, ${ }^{1,2}$ Arifah Bahar ${ }^{*}$ and ${ }^{2,4}$ Zainal Abdul Aziz \\ ${ }^{1}$ Department of Mathematical Sciences, Faculty of Science \\ Universiti Teknologi Malaysia, 81310 Johor Bahru, Johor, Malaysia \\ ${ }^{2}$ Centre for Industrial and Applied Mathematics, \\ Universiti Teknologi Malaysia, 81310 Johor Bahru, Johor, Malaysia \\ ${ }^{3}$ Faculty of Computer and Mathematical Sciences \\ Universiti Teknologi MARA, 40450 Shah Alam, Selangor, Malaysia \\ ${ }^{4}$ MYHIMS SOLUTIONS PLT, \\ Level 3, C08, Universiti Teknologi Malaysia, 81310 Johor Bahru, Johor, Malaysia
}

*Corresponding author: arifah@utm.my

Article history

Received: 22 August 2018

Received in revised form: 6 December 2018

Accepted: 17 December 2018

Published on line: 31 December 2018

\begin{abstract}
The flow of water over an obstacle is a fundamental problem in fluid mechanics. Transcritical flow means the wave phenomenon near the exact criticality. The transcritical flow cannot be handled by linear solutions as the energy is unable to propagate away from the obstacle. Thus, it is important to carry out a study to identify suitable model to analyse the transcritical flow. The aim of this study is to analyse the transcritical flow over a bump as localized obstacles where the bump consequently generates upstream and downstream flows. Nonlinear shallow water forced Korteweg-de Vries (fKdV) model is used to analyse the flow over the bump. This theoretical model, containing forcing functions represents bottom topography is considered as the simplified model to describe water flows over a bump. The effect of water dispersion over the forcing region is investigated using the fKdV model. Homotopy Analysis Method (HAM) is used to solve this theoretical fKdV model. The HAM solution which is chosen with a special choice of $\hbar$-value describes the physical flow of waves and the significance of dispersion over a bump is elaborated.
\end{abstract}

Keywords Approximate analytic solution; fKdV equation; $\hbar$-curve; homotopy analysis method; transcritical flow.

Mathematics Subject Classification 76B15, 35Q53.

\section{Introduction}

The flow of water over an obstacle is a fundamental problem in fluid mechanics. Generation of solitary waves by bottom forcing topography has gained attention since the experimental research by Huang et al. [1] and numerical findings by $\mathrm{Wu}$ and $\mathrm{Wu}$ [2]. 
Linear theory is used to describe the wave field when the flow is not critical. Critical in this context means the flow speed is not close to any linear long wave speed. These linear theory solutions only applicable to the subcritical case (flow speed <long wave speed) or supercritical (flow speed >long wave speed). This identifies that the linear solutions fail near criticality condition (flow speed $\approx$ long wave speed) as the energy is unable to propagate away from the obstacle [3]. Thus it is crucial to identify a suitable model to analyse the wave phenomenon near the exact criticality or transcritical flow. It is found that the forced Korteweg-de Vries $(\mathrm{fKdV})$ equation is a simpler theoretical model to study the free surface flow over a bump [3].

The standard form of $\mathrm{fKdV}$ equation for water waves is given by Pelinovsky et al. [4],

$$
\eta^{\prime}(t)+\Delta \eta^{\prime}(x)+\alpha \eta \eta^{\prime}(x)+\beta \eta^{\prime \prime \prime}(x)=f^{\prime}(x)
$$

with $\alpha=3 c / 2 h_{0}, \beta=c h_{0}^{2} / 6$ and $f=-c z / 2$

where $\eta$ refers to the elevation of free water surface, $z$ represents the solid bottom, $h$ is the constant mean water depth, $c$ is the long wave speed with $\mathrm{g}$ is acceleration due to gravity, and $\Delta=$ critical parameter.

Forced KdV equation with various types of forcing term have been studied since 30 years ago by Patoine and Warn [5], Akylas [6], Grimshaw and Smyth [7], Wu [8], and Lee et al. [9]. Lee et al. [9] found that fKdV model admits external forcing disturbances when the surface pressure and bottom topography are entirely equivalent. Grimshaw and Smyth [7] showed that the forcing term of fKdV equation was characterised by forcing amplitude and length scale forcing positive parameter. Length of disturbance may have a crucial effect on solitary wave generation as was highlighted by Shen [10]. The effect of forcing length on wave amplitude was studied by Teng and $\mathrm{Wu}$ [11]. Zhang and Zhu [12] presented a nonlinear theory for different ranges of Froude numbers varying from subcritical, transcritical and supercritical. Zhang and Chwang [13] studied the generation of solitary waves at the critical velocity on different bottom topographies. Grimshaw et al. [3] explored transcritical flow using fKdV model where numerical and asymptotic analytical solutions have shown upstream and downstream flows.

The Homotopy Analysis Method (HAM) is an analytical method to solve nonlinear partial differential equations, which was first introduced by Liao [14]. HAM has greater flexibility in the selection of a proper set of base functions for the solution and a much simpler way in the control of the convergence rate and region compared to perturbation approach [ $[15$, $16,17,18]$. This analytical technique does not have restriction of non-perturbation methods, such as Lyapunovs artificial small parameter method, the $\delta$-expansion method and Adomian's decomposition method. The analytical technique also has been applied successfully in many nonlinear problems in engineering and sciences [19].

For example, in the nonlinear progressive waves [20], free oscillations of positively damped systems with algebraically decaying amplitude [21], free oscillations of self-excited systems [22] and similarity boundary layer equations [23]. The HAM is applied to obtain the solitary solution of $\mathrm{KdV}$ equation and it shows excellent agreement with the exact solution [24]. Up to now, solutions of fKdV equation can only be obtained by numerical or perturbation series [25]. Recently, fKdV model with a specific choice of forcing term is successfully solved using HAM $[26]$.

In this paper, the flow over a bump will be analysed using shallow water fKdV model. Shallow water fKdV model will be solved using HAM. The fKdV model has a forcing term which represents the bottom topography. This investigation aims (a) to describe the localized 
obstacle or bump (b) to find an analytical approximate solution for fKdV using a forcing term that represents the bump (c) to explore and analyse the flow over the bump physically, and (d) to describe the effect of dispersion over the forcing region. It is found that HAM solution could explain the flows of water over a bump. The bump is found to generate upstream and downstream flows. The bump also creates a uniform depth wave over the forcing region.

\section{Methodology}

\subsection{Shallow Water fKdV with Bottom Topography}

Rewrite the Equation (1) in dimensional form, Wu [8],

$$
\left(1 / c_{0}\right) \phi^{\prime}(t)+\left[(F r-1)-(3 / 2)\left(\phi / h_{0}\right)\right] \phi^{\prime}(x)-(1 / 6) h_{0}^{2} \phi^{\prime \prime \prime}(x)=(1 / 2) f^{\prime}(x)
$$

where $\varphi$ refers to the water elevation, $f$ for forcing term, $F r$ and Froude number and $c_{0}$ for linear long wave speed.

It should be noted that the Equation (2) is a nonlinear shallow water fKdV which is derived from the theory of fluid mechanics [27]. The term $\Delta=(F r-1)$ is the criticality parameter where it is classified into three types of critical flow according to the value of $F r$. The three types of critical flow are transcritical $(\Delta=0)$, subcritical $(\Delta<0)$ and supercritical $(\Delta>0)$. The right hand side of Equation (2) represents the forcing term. The relation of the forcing term and seabed geometry can be seen in Equation (2).

\subsection{HAM on fKdV}

We attempt to solve Equation (2) for the transcritical flows. Generalizing Equation (2),

$$
\alpha \phi^{\prime}(t)+\beta \phi^{\prime}(x)+\lambda \phi \phi^{\prime}(x)+\sigma \phi^{\prime \prime \prime}(x)+\varpi f^{\prime}(x)=0
$$

where $\alpha=1 / c_{0}, \beta=F r-1=\left(U / c_{0}\right)-1 \approx 0, \lambda=-3 / 2 h_{0}, \sigma=-h_{0}^{2} / 6, \varpi=-0.5 f_{m}$

Consider the constant mean water depth, $h_{0}=1$ unit, wave speed, $c_{0} \approx c \approx \sqrt{g h}=\sqrt{9.81}$, maximum height of topography chosen, $f_{m}=0.1$ and forcing term, $f=-z / 2$ where $z=$ $f_{m} \exp \left(-x^{2} / 4\right)$. Figure 1 is the sketch of the bottom topography, $z$.

From HAM,

$$
(1-q) \ell\left[\phi(x, t ; q)-\phi_{0}(x, t)\right]=q \hbar \mathcal{H}(x, t) N[\phi(x, t ; q)]
$$

we use $\phi_{0}(x, t)=(1+\sin [x]) / 4$

as the initial guess and

$$
\ell[\phi(x, t ; q)]=\frac{\partial \phi(x, t ; q)}{\partial t}
$$

as the auxiliary linear operator satisfying

$$
\ell[c]=0
$$

where $c$ is constant.

Considering

$$
\mathcal{H}(x, t)=1
$$




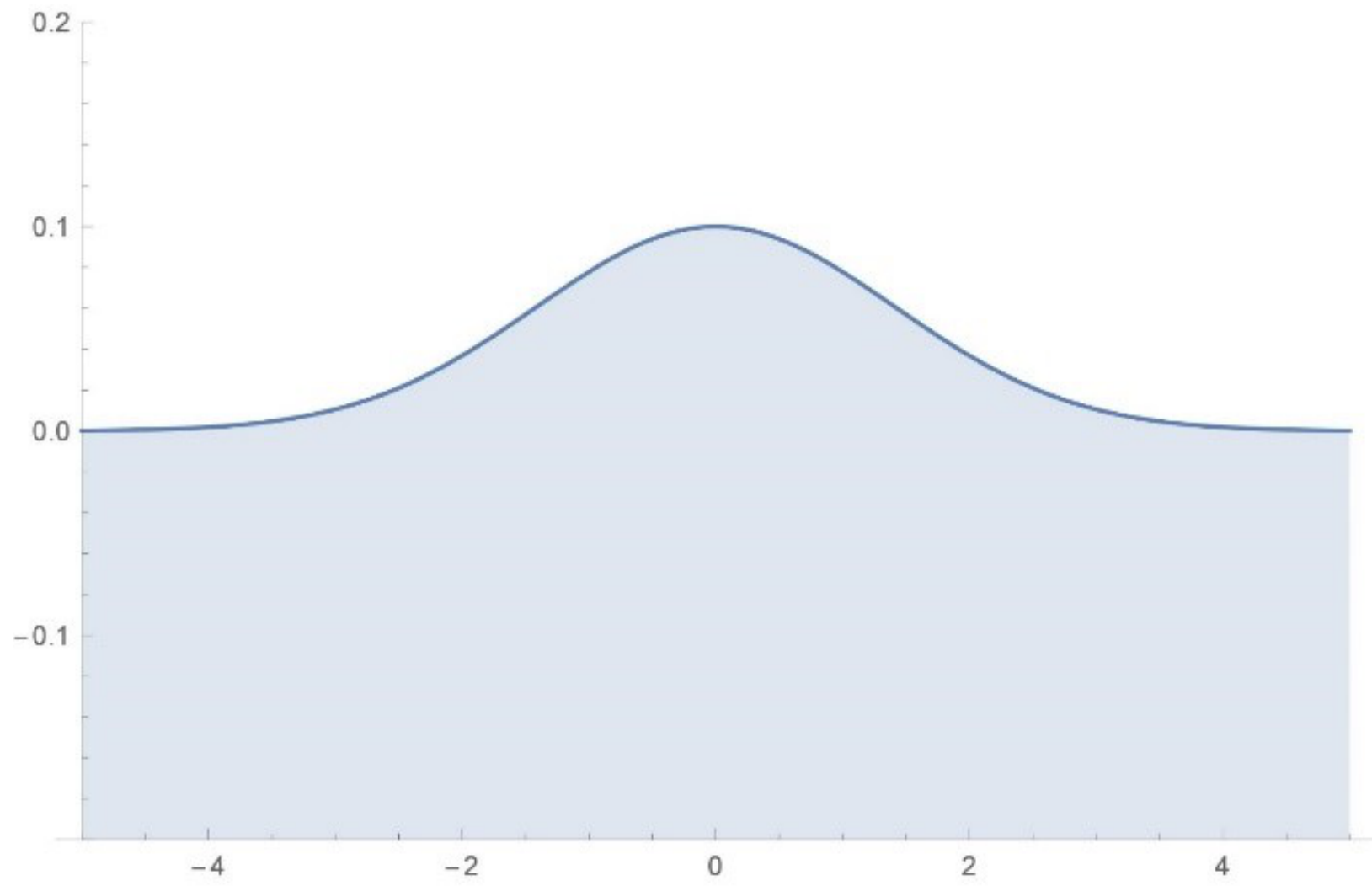

Figure 1: Bottom Topography, $z$ for $z=0.1 \exp \left(-x^{2} / 4\right)$

$$
N[\phi(x, t ; q)]=\alpha \frac{\partial \phi(x, t ; q)}{\partial t}+\beta \frac{\partial \phi(x, t ; q)}{\partial x}+\lambda \phi(x, t ; q) \frac{\partial \phi(x, t ; q)}{\partial x}+\sigma \frac{\partial^{3} \phi(x, t ; q)}{\partial x^{3}}+\varpi \frac{\partial f}{\partial x}
$$

and the $m$ th-order deformation problem

$$
\begin{aligned}
& \ell\left[\phi_{m}(x, t)-\chi_{m} \phi_{m-1}(x, t)\right]= \\
& q \hbar\left[\alpha \frac{\partial \phi_{m-1}}{\partial t}+\beta \frac{\partial \phi_{m-1-i}}{\partial x}+\lambda\left(\sum_{i=0}^{m-1} \phi_{i} \frac{\partial \phi_{m-1-i}}{\partial x}\right)+\sigma \frac{\partial^{3} \phi_{m-1}}{\partial x^{3}}+\varpi \frac{\partial f_{m-1}}{\partial x}\right]
\end{aligned}
$$

with

$$
\phi_{m}(x, 0)=0 \text { for } m>1
$$

\section{Result and Discussion}

\subsection{HAM Solution of Transcritical Flow}

In the case of transcritical flow, we let $F r=1$. MATHEMATICA Version 10 was used to solve the nonlinear equations. HAM solution of Equation (2) is obtained at 6th-order approximation. The solution is

$$
\phi(x, t)=\phi_{0}(x, t)+\phi_{1}(x, t)+\ldots+\phi_{6}(x, t)
$$




$$
\begin{aligned}
\phi(x, t) & =\frac{1}{4}(1+\sin x)+\frac{1}{16} e^{-\frac{x^{2}}{4}} \hbar t\left(\frac{2 x}{5}-\frac{5}{6} e^{\frac{x^{2}}{4}} \cos x-\frac{3}{2} e^{\frac{x^{2}}{4}} \cos x \sin x\right) \\
& +\frac{1}{512} e^{-\frac{x^{2}}{4}} \hbar\left(\frac{64 t}{5}-\frac{4 \hbar t^{2}}{5}+\frac{64 t x}{5}+4.08673 \hbar t x-\frac{32 t x^{2}}{5}-\frac{2}{5} \hbar t^{2} x^{2}+\frac{2}{15} \hbar t^{2} x^{4}\right. \\
& -\frac{80}{3} e^{\frac{x^{2}}{4}} t \cos x-8.51401 e^{\frac{x^{2}}{4}} \hbar t \cos x-\frac{12}{5} \hbar t^{2} x \cos x-2 e^{\frac{x^{2}}{4}} \hbar t^{2} \cos (2 x) \\
& -\frac{12}{5} \hbar t^{2} \sin x-\frac{181}{36} e^{\frac{x^{2}}{4}} \hbar t^{2} \sin x+\frac{6}{5} \hbar t^{2} x^{2} \sin x-\ldots .
\end{aligned}
$$

In order to obtain a better convergence of the HAM solutions, the value of $\hbar$ is determined by plotting the derivatives of $\phi$ for a fixed point of $x$ and time, $t$. Figure 2 shows the $\hbar$-curves at 6 th order approximation.

The relation of the forcing term and seabed geometry can be seen in Equation (2). It is pointed out that the valid region of $\hbar$ lies on the horizontal line segment. The admissible convergence interval of HAM solution is $-5 \leq \hbar \leq 0.5$. After a detailed computation using MATHEMATICA Version $10, \hbar$ is determined at -2 .

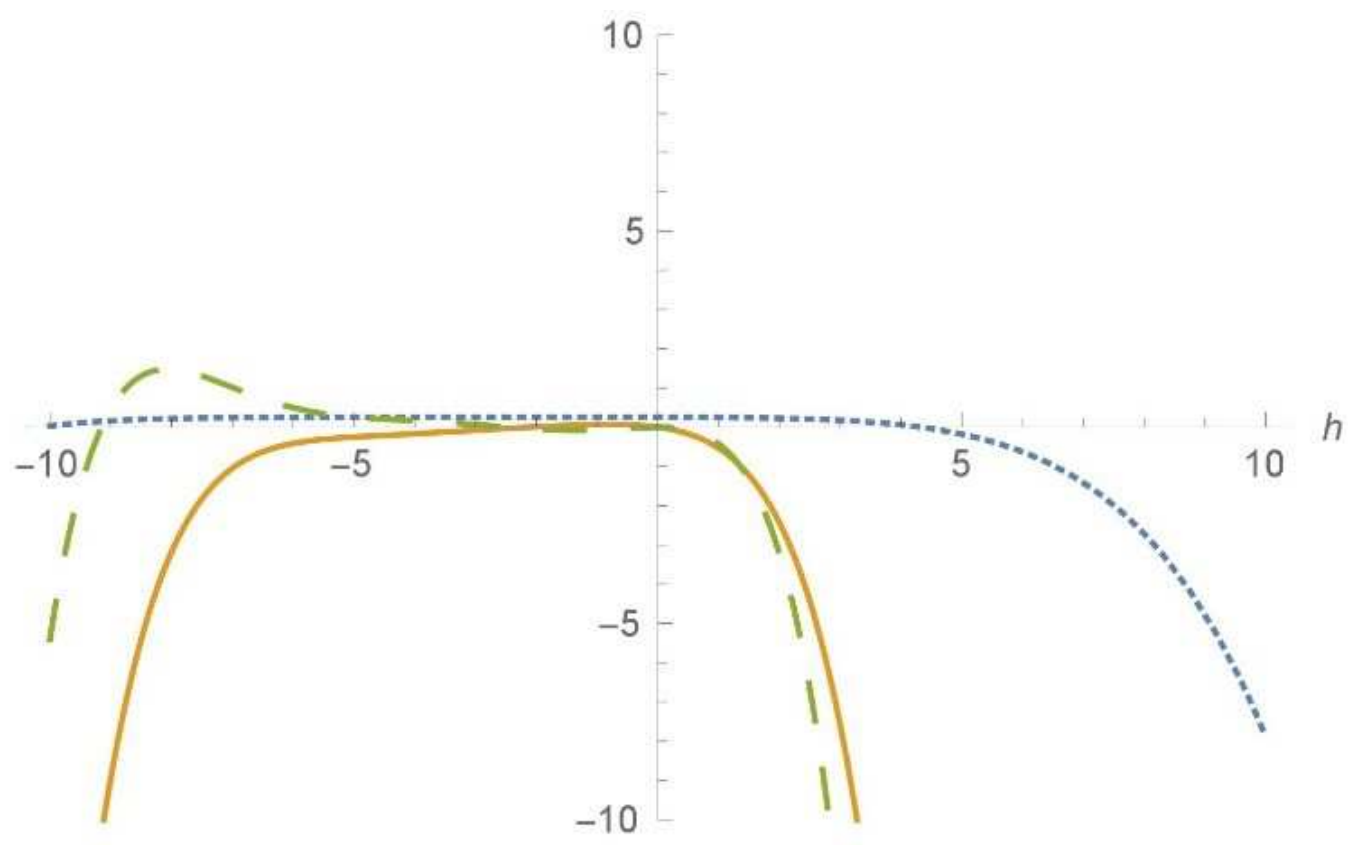

Figure 2: The $\hbar$-curves According to the 6th Order Approximation. $\phi(0.01,0.01), \phi \bullet(0.01,0.01)$, $\phi \bullet \bullet(0.01,0.01)$

Figure 3 shows the flow of waves over a bump at $t=5$. The bottom line in Figure 3 is the sketch of bump with the height of 0.1 units at the point $x=0$. Figure 4 displays the flow over a bump where the bump continuously generates the upstream and downstream flows. The HAM solution of the Figures 3 and 4 can be split into 3 stages. The first stage is upstream flow over the region of $x<0$ continued by the second stage of flow over the bump $(0<x<2.5)$ and finally, the downstream flow at $x>2.5$. 


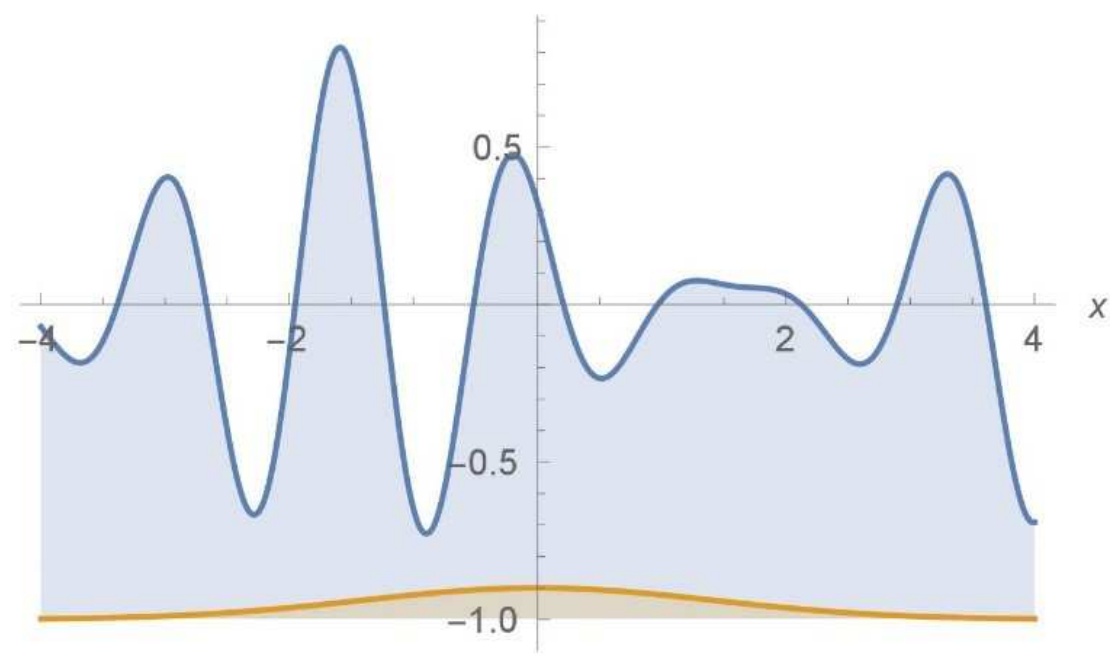

Figure 3: Plot of HAM Solution for Equation (4) at $t=5$. Bottom Line: Bump, $z$ and Upper Line: Water Elevation, $\phi(x, 5)$

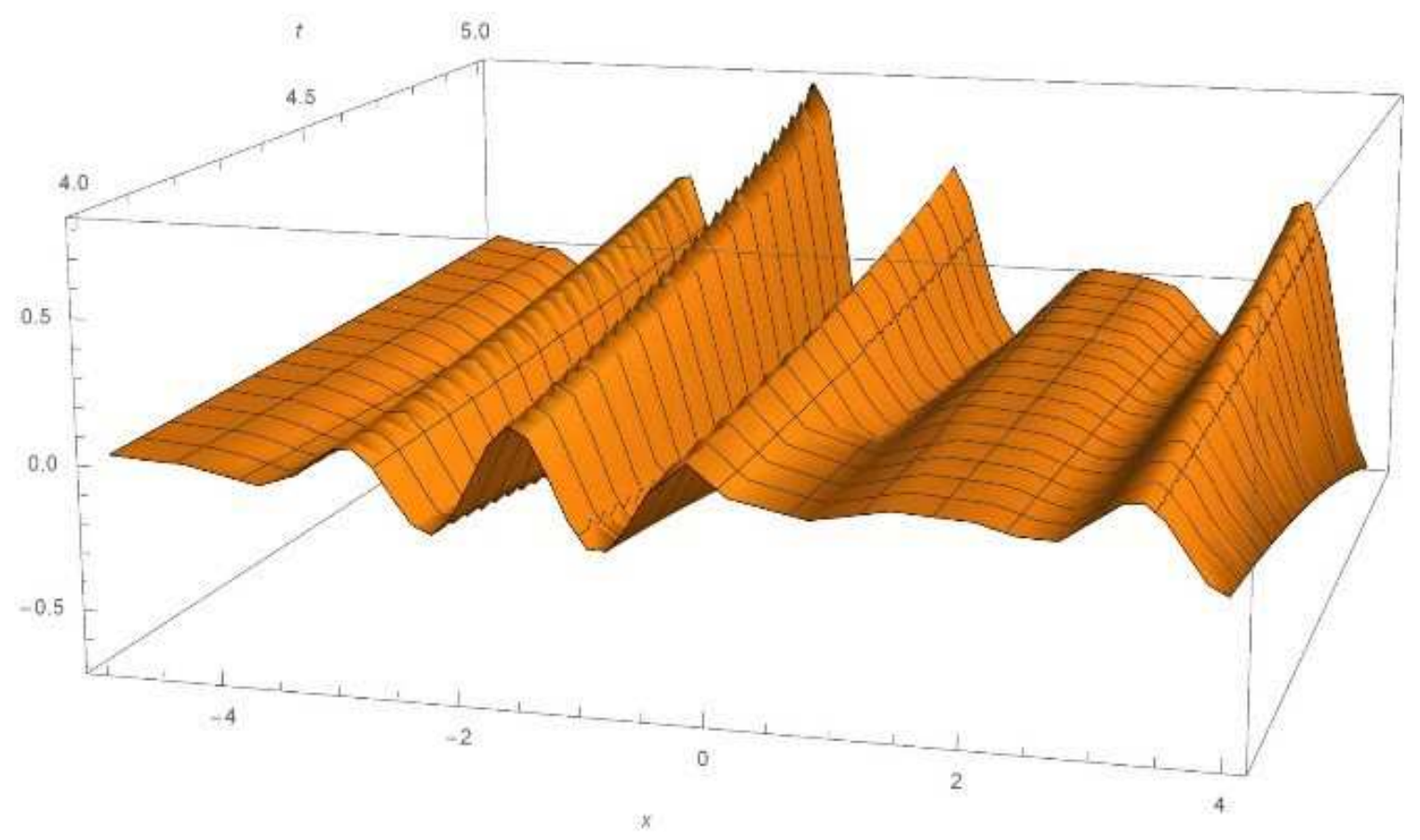

Figure 4: Three Dimensional Plot of HAM Solution for Equation (4) for $4 \leq \mathrm{t} \leq 5$ 
Many researches have been done on flow over the bump by omitting the dispersive term. Grimshaw and Smyth found that the effect of dispersion is significant in the forcing region as well as in the formation of waves upstream and downstream [7]. In the case of weak dispersion, it may cause a formation of a solitary-type wave which propagates upstream. Grimshaw and Smyth [7], also stated that in the case of strong dispersion, the fKdV solution may reach the quasi-stationary state in the forcing region and the downstream depression terminates. Then each solitary wave that propagates from upstream will flow smoothly over the bump so that the wave will be sent downstream.

In this work, the dispersive term is taken into consideration using fKdV model. At the initial stage, solitary waves are successively generated at the point of $\mathrm{x}<0$. We can see that the upstream flows are attached to the bump and there is a sequence of wavetrains generated towards upstream from the bump. The sinusoidal function is chosen as the initial guess function (Equation (7)). This suggests that the water waves flow over in wave form from the left to the right. So, when the water waves collide the bump, there exists a resonant flow opposite to the current flow. This indicates that the bump generates upstream flows towards the left through the act of dispersion. This act of dispersion is much similar with Grimshaws [7] findings in 1986. It should be noted that the flow of upstream and downstream are not connected at a point where we can notice that there is no communication between the two ends of the steps. We can clearly see that the waves are horizontally flattened in the coordinate of $1 \leq x \leq 2.2$. Grimshaw and Smyth [7], and $\mathrm{Wu}[8]$ observed the similar horizontally flattened of water waves in their work. This suggests that the effect of dispersion is weak, yet it still plays an important role in the flow over a bump. Referring to Figure 4, the period of waves is similar but it has different amplitudes of the upstream waves. HAM solution depicts that the amplitude of the waves at upstream is increasing slowly over time. Existing numerical solutions for flow over a bump suggested that the amplitude of the wave upstream is stationary [3]. However, available numerical solutions as in Grimshaw and Smyth [7], and $\mathrm{Wu}[8]$ show that the wave on bump has a lower height compare to earlier waves. This seems to be agreeable with HAM solution where the waves on the bump has a height of 0.5 units and the earlier waves of upstream is about 0.8 units.

At the second stage of the flow over a bump, the amplitude of the generated solitary waves reduces after it flows over the peak of bump at $x=0$. It does not produce solitary waves immediately but produce a uniform depth of flows. It can be seen from the figures 3 and 4 that, the waves are flattened horizontally for $1<x<2.2$. Finally, at the downstream $(x>$ $2.5)$, a cnoidal-like waves start oscillating. Solitary waves are observed at the downstream.

\section{Conclusion}

In this work, the free surface transcritical flow over a bump has been observed using a shallow water forced Korteweg-de Vries (fKdV) model. FKdV model is well-known as the simplified models to analyse the flow over a bump. Bump has been used as a forcing region to identify the forcing term of fKdV. An analytical approximate solution for shallow water fKdV equation using HAM is obtained. Horizontal line segment with the $\hbar$-curves strongly shows that the obtained analytical approximate solution is fully converged. A logical and suitable HAM solution is obtained by using a coefficient, $k$. The obtained HAM solutions show that when the water flows over the bump, the bump consequently generates upstream flows which then creates 
uniform depth waves over the forced region and finally generate oscillatory downstream flows. The behaviour of water flow obtained in this research is similar and agreeable with the work done in Grimshaw (1986). Even though, the dispersion of the free surface transcritical flow is found to be weak in this model, it should be noted that the effect of dispersion still plays an important role in generating the upstream flows.

\section{Acknowledgement}

The first author is thankful to Universiti Teknologi MARA, UiTM for the educational scholarship.

\section{References}

[1] Huang, D. B., Sibul, O. J., Webster, W. C., Wehausen, J. V., Wu, D. M., and Wu, T. Y. Ships moving in the transcritical range. In Proceedings Conference on Behaviour of Ships in Restricted. Waters, Varna, Bulgaria. 1982. 26-1.

[2] Wu, D. M., and Wu. T.Y. Three-dimensional nonlinear long waves due to moving surface pressure. In Proceedings of the 14th Symposium on Naval Hydrodynamics. 1982. 103-129.

[3] Grimshaw, R. H., Zhang, D. H., and Chow, K.W. Generation of solitary waves by transcritical flow over a step. Journal of Fluid Mechanics. 2007. 587: 235-254.

[4] Yalçiner, A. C., Pelinovsky, E. N., Okal, E., and Synolakis, C. E. Submarine Landslides and Tsunamis. Springer Science and Business Media. 2012. (21): 111.

[5] Patoine, A., and Warn, T. The interaction of long, quasi-stationary baroclinic waves with topography. Journal of the Atmospheric Sciences. 1982. 39(5): 1018-1025.

[6] Akylas, T.R. On the excitation of long nonlinear water waves by a moving pressure distribution. Journal of Fluid Mechanics. 1984. 141: 455-466.

[7] Grimshaw, R. H., and Smyth, N. Resonant flow of a stratified fluid over topography. Journal of Fluid Mechanics. 1986. 169: 429-464.

[8] $\mathrm{Wu}, \mathrm{T}$. Generation of upstream advancing solitons by moving disturbances. Journal of Fluid Mechanics. 1987. 184: 75-99.

[9] Lee, S. J., Yates, G. T., and Wu, T. Y. Experiments and analyses of upstream-advancing solitary waves generated by moving disturbances. Journal of Fluid Mechanics. 1989. 199: 569-593.

[10] Shen, S. S. Forced solitary waves and hydraulic falls in two-layer flows. Journal of Fluid Mechanics. 1992. 234: 583-612.

[11] Teng, M. H., and Wu, T. Y. Effect of disturbance length on resonantly forced nonlinear shallow water waves. International Journal of Offshore and Polar Engineering. 1997. 7(04).

[12] Zhang, Y. and Zhu, S. Subcritical, transcritical and supercritical flows over a step. Journal of Fluid Mechanics. 1997. 333: 257-271.

[13] Zhang, D. H. and Chwang, A. T. Generation of solitary waves by forward-and backwardstep bottom forcing. Journal of Fluid Mechanics. 2001. 432: 341-350. 
[14] Liao, S. J. The Proposed Homotopy Analysis Technique for The Solution of Nonlinear Problems. Ph. D. Thesis. Shanghai Jiao Tong University. 1992.

[15] Liao, S. J. Beyond Perturbation: Introduction to The Homotopy Analysis Method. CRC press. 2003.

[16] Liao, S. J. Comparison between the homotopy analysis method and homotopy perturbation method. Applied Mathematics and Computation. 2005. 169(2): 1186-1194.

[17] Liao, S. J. and Tan, Y. A general approach to obtain series solutions of nonlinear differential equations. Studies in Applied Mathematics. 2007. 119(3): 297-354.

[18] Liao, S. J. Notes on the homotopy analysis method: some definitions and theorems. Communications in Nonlinear Science and Numerical Simulation. 2009. 14(4): 983-997.

[19] Abbasbandy, S. The application of homotopy analysis method to solve a generalized Hirota-Satsuma coupled KdV equation. Physics Letters A. 2007. 361(6): 478-483.

[20] Liao, S. J. and Cheung, K. F. Homotopy analysis of nonlinear progressive waves in deep water. Journal of Engineering Mathematics. 2003. 45(2): 105-116.

[21] Liao, S. J. An analytic approximate technique for free oscillations of positively damped systems with algebraically decaying amplitude. International Journal of Non-Linear Mechanics. 2003. 38(8): 1173-1183.

[22] Liao, S. J. An analytic approximate approach for free oscillations of self-excited systems. International Journal of Non-Linear Mechanics. 2004. 39(2): 271-280.

[23] Liao, S. J. and Pop. I. Explicit analytic solution for similarity boundary layer equations. International Journal of Heat and Mass Transfer. 2004. 47(1): 75-85.

[24] Nazari, M., Salah, F., Abdul Aziz, Z., and Nilashi, M. Approximate analytic solution for the KdV and Burger equations with the homotopy analysis method. Journal of Applied Mathematics. 2012.

[25] Jun-Xiao, Z. and Bo-Ling. G. Analytic solutions to forced KdV equation. Communications in Theoretical Physics. 2009. 52(2): 279.

[26] David, V. D., Nazari, M., Barati, V., Salah, F. and Abdul Aziz, Z. Approximate analytical solution for the forced Korteweg-de Vries equation. Journal of Applied Mathematics. 2013.

[27] Shen, S. S. A Course on Nonlinear Waves. Springer. 1993. 